\title{
A Green Antioxidant Activity-Integrated Dual-Standard Method for Rapid Evaluation of the Quality of Traditional Chinese Medicine Xuebijing Injection by On-Line DPPH-CE-DAD
}

\author{
Jin Li, ${ }^{1}$ Jiao Liu, ${ }^{1}$ Wei Liu, ${ }^{1}$ Xie-an Yu, ${ }^{1}$ Jun Cao, ${ }^{2}$ Mingrui An, ${ }^{3}$ \\ Xiu-mei Gao, ${ }^{1}$ and Yan-xu Chang ${ }^{1}$ \\ ${ }^{1}$ Tianjin State Key Laboratory of Modern Chinese Medicine, Tianjin University of Traditional Chinese Medicine, Tianjin 300193, China \\ ${ }^{2}$ College of Material Chemistry and Chemical Engineering, Hangzhou Normal University, Hangzhou 310036, China \\ ${ }^{3}$ Department of Surgery, University of Michigan, Ann Arbor, MI 48109, USA
}

Correspondence should be addressed to Jun Cao; caojun91@163.com and Yan-xu Chang; tcmcyx@126.com

Received 26 July 2016; Accepted 30 August 2016

Academic Editor: Fabio Firenzuoli

Copyright ( 92016 Jin Li et al. This is an open access article distributed under the Creative Commons Attribution License, which permits unrestricted use, distribution, and reproduction in any medium, provided the original work is properly cited.

\begin{abstract}
Much attention has been focused on treatment of sepsis which leads to high mortality all over the world in every year. Antioxidant activity seems to play a prominent role in the treatment of sepsis exhibited by Xuebijing injection. The aim of the present research was to develop an on-line 1, 1-diphenyl-2-picrylhydrazyl- (DPPH-) capillary electrophoresis-diode array detector (on-line DPPH-CEDAD) method for rapidly assessing antioxidant properties and efficacious material basis of antioxidant activity as a way of quality control of Xuebijing injection. Several parameters affecting the separation were investigated, including the $\mathrm{pH}$ and concentrations of buffer, SDS, $\beta-\mathrm{CD}$, and organic modifier as well as voltage and cassette temperature. Compared to previous traditional method, this improved method shortened the experimental cycle and became more efficient because it was successfully applied to analyze total antioxidant activity and contents of twelve antioxidants of Xuebijing injection under the same condition. The results revealed that the on-line DPPH-CE-DAD method was a reagent-saving, rapid, feasible, and green technique for quality control of Xuebijing injection in terms of pharmacological activity and contents of active ingredients. It also offered new opportunities for the analysis of antioxidant activity of complex matrix.
\end{abstract}

\section{Introduction}

Sepsis, a systemic inflammatory response syndrome caused by infection, is confirmed to be accompanied with the presence of bacteria or highly suspicious focus of infection [1]. Despite the use of antibiotic combination and good supportive therapy and care, treatment for sepsis is still unsatisfactory. The mortality from severe sepsis remains high between 34 and 43\% [2]. In a recent septic immunomodulatory study, the traditional Chinese medicine (TCM) attracted much attention for its therapeutic concepts of integration and balanced regulation [3]. Xuebijing (XBJ) injection, extracted from Carthami flos, Paeoniae Radix Rubra, Chuanxiong Rhizoma, Salviae miltiorrhizae, and Angelicae sinensis Radix, is a traditional Chinese medicine that has been approved for many years by the State Food and Drug Administration (SFDA) of China to clinically treat sepsis [4]. Recent studies has also shown that XBJ is effective for the treatment of serious complications of sepsis, such as hematopoietic injury [4], disseminated intravascular coagulation [5], hematopoietic injury [6], and lung injury [1]. It has been reported that oxidative stress was caused by the pathological process of viral infection and antioxidants could reduce oxidative stress $[7,8]$. It has also been demonstrated that Xuebijing injection could decrease the levels of reactive oxygen species (ROS) by increasing glutathione and superoxide dismutase (SOD) levels [4]. Thus, it can be speculated that Xuebijing injection possesses antioxidant properties. However, the underlying 
material basis of antioxidant activity of Xuebijing injection remains uninvestigated.

Traditional methods of quality control of TCMs included High-Performance Liquid Chromatography (HPLC), Gas Chromatography-Mass Spectrometry (GC-MS), and HPLCMS [9-11]. Because these methods are limited in determining the contents of compounds, they cannot meet our needs to comprehensively assess the quality of TCMs expected in combination for their pharmacological effect [12]. Therefore, the dual-standard quality assessment was introduced in this study to establish a simple and feasible method to screen the antioxidant components and evaluate the quality of Xuebijing injection.

1, 1-Diphenyl-2-picrylhydrazyl (DPPH) which is a radical-containing compound is usually used to quantify the antioxidant activity of various samples $[13,14]$. Various methods were used for the assessment of antioxidants in complex samples, such as the DPPH radical with a spectrophotometer, dot-blot test on a thin-layer chromatography (TLC) plate, and on-line HPLC-DPPH assay [14-16]. These pharmacological methods can lead to long cycle, false positives and can be harmful to the environment. Recently, high-performance capillary electrophoresis (HPCE), as a green separation technology, offers an alternative method that has many advantages compared to other methods, in that it provides good resolution of the sample with a shorter run time, uses less harmful solvents, and is not influenced by color pigments $[17,18]$.

In a previous paper, we reported our preliminary finding that the on-line DPPH-CE-DAD conditions for determination of the total antioxidant activity and contents of antioxidants were entirely different [18]. If two conditions can be integrated into the same condition, thus the total time needed for the experiment will be significantly cut down and less intensive labor will be achieved. Taking this into account, we have developed a single method that permits the separation and quantification of DPPH and the antioxidants under the same condition by integrating two separation methods into one step. The on-line DPPH-capillary electrophoresisdiode array detector (on-line DPPH-CE-DAD) in a run was selected as a typical example to develop a simple and feasible dual-standard method to screen the antioxidant components and evaluate the quality of Xuebijing (XBJ) injection. The feasibility and precision of on-line DPPH-CE-DAD were discussed in our present report. Schematic diagram of online DPPH-CE-DAD method was shown in Figure 1. The improved DPPH-CE-DAD method provided a green, environmental protection and rapid approach to quantitatively analyze total antioxidant activity and contents of antioxidants of Xuebijing injection and other TCMs in short time. An antioxidant activity-integrated dual-standard method by online DPPH-CE-DAD will become the advantageous tool for quality control of TCMs.

\section{Materials and Methods}

2.1. Chemicals and Reagents. Standard substances including oxypaeoniflorin, hydroxysafflor yellow A, protocatechuic aldehyde, peoniflorin, rosmarinic acid, salvianolic acid B, sodium danshensu, caffeic acid, ferulic acid, senkyunolide I, rutin, and isoquercitrin were purchased from Chengdu Must Bio. Sci. and Tec. Co. Ltd. (Chengdu, China). 10 batches of Xuebijing injections were obtained from Tianjin Chase Sun Pharmaceutical Co. Ltd. (Tianjin, China). Deionized water was provided by a Milli-Q Academic ultra-pure water system (Millipore, Milford, MA, USA). HPLC grade methanol and acetonitrile were obtained from Merck (Germany). DPPH (1, 1-diphenyl-2-picrylhydrazyl) was purchased from Sigma (USA). Other chemicals were of analytical reagent grade. All reagents for capillary electrophoresis were filtrated through $0.22 \mu \mathrm{m}$ nylon syringe filter.

2.2. Preparation of Standard Solutions. Oxypaeoniflorin, hydroxysafflor yellow A, protocatechuic aldehyde, peoniflorin, rosmarinic acid, salvianolic acid B, and sodium danshensu were individually dissolved with deionized water. Caffeic acid, ferulic acid, rutin, and isoquercitrin were individually dissolved with 50\% methanol. Senkyunolide I was dissolved with methanol. Appropriate amount of the standards was mixed to prepare a standard solution containing twelve compounds. DPPH solution was dissolved with methanol at a concentration of $1 \mathrm{mg} / \mathrm{mL}$ for each day of analysis and stored in the dark prior to use. Standard solutions and samples were stored at $4^{\circ} \mathrm{C}$.

2.3. Preparation of Quality Control Samples. Quality control (QC) samples of oxypaeoniflorin, hydroxysafflor yellow A, protocatechuic aldehyde, peoniflorin, rosmarinic acid, salvianolic acid B, sodium danshensu, caffeic acid, ferulic acid, senkyunolide I, rutin, and isoquercitrin were prepared by diluting appropriate mixed standard solutions to make three concentration levels (low, medium, and high), respectively.

2.4. Preparation of Samples. The Xuebijing injection samples were centrifuged at $14,000 \mathrm{rpm}$ for $10 \mathrm{~min}$. Supernatant was then filtrated through $0.22 \mu \mathrm{m}$ nylon prior to injection. The different concentration of Xuebijing injection was diluted with deionized water and analyzed by HPCE.

2.5. Apparatus and Conditions of DPPH-CE-DAD Method. Capillary electrophoresis was carried out on an Agilent technologies HPCE 7100 (Agilent, Germany) equipped with a diode array detector and a sample tray temperature control system. Agilent ChemStation software for instrumental control and data processing was used. The analysis was performed on an uncoated capillary with effective length of $52 \mathrm{~cm}$ and an internal diameter of $50 \mu \mathrm{m}$ (Ruifeng, Hebei, China). Before the new capillary was initiated, it was flushed with $1.0 \mathrm{M} \mathrm{NaOH}$ for $10 \mathrm{~min}$, followed by $0.1 \mathrm{M} \mathrm{NaOH}$ for $10 \mathrm{~min}$ and deionized water for $10 \mathrm{~min}$. In between each sample throughout the experiment, the capillary was rinsed with $0.1 \mathrm{M} \mathrm{NaOH}$ for $3 \mathrm{~min}$, deionized water for $3 \mathrm{~min}$, and buffer for $3 \mathrm{~min}$, successively. The electrolyte buffer was a solution containing $20 \mathrm{mM} \mathrm{NaH} \mathrm{PO}_{4}(\mathrm{pH} 5.5), 100 \mathrm{mM}$ sodium dodecyl sulfate (SDS), $10 \mathrm{mM} \beta$-cyclodextrin $(\beta$-CD), and $5 \% \operatorname{ACN}(\mathrm{v} / \mathrm{v})$. To estimate the total antioxidant activity and 

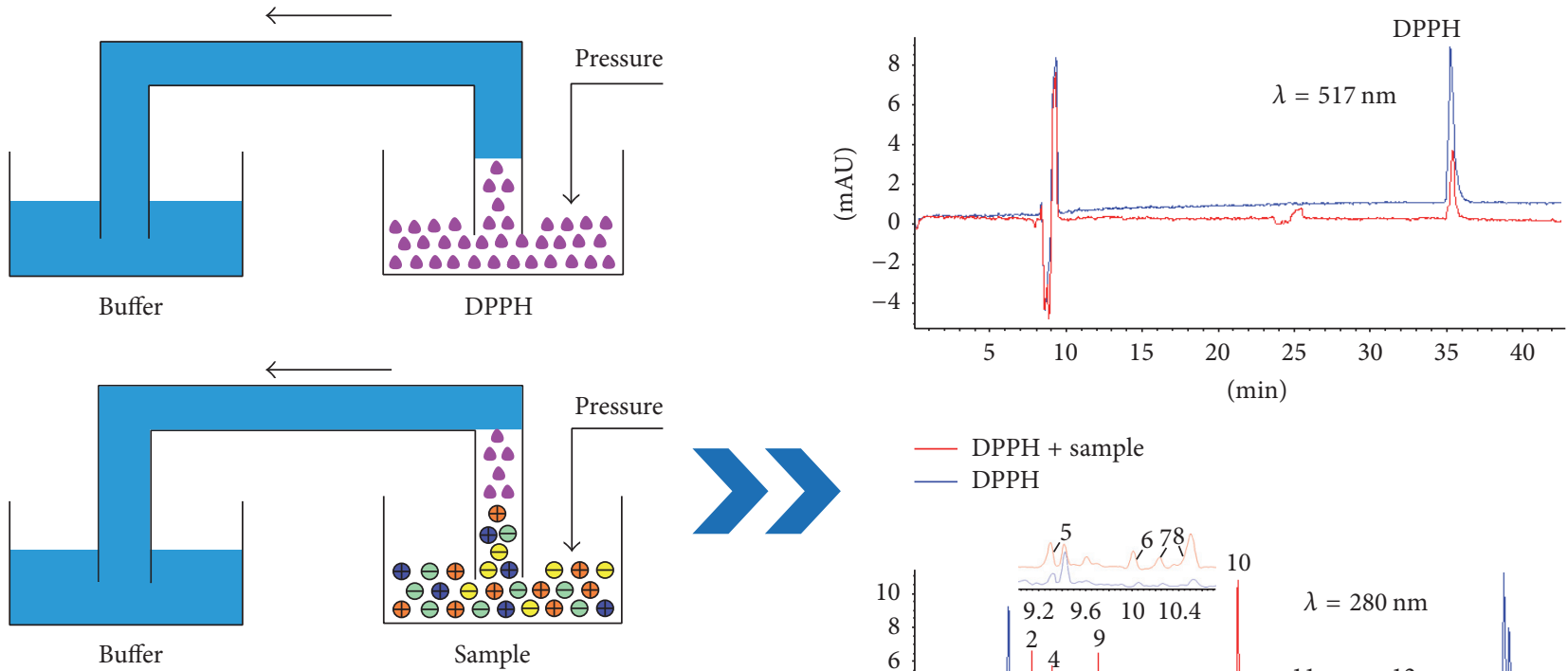

— DPPH + sample - DPPH

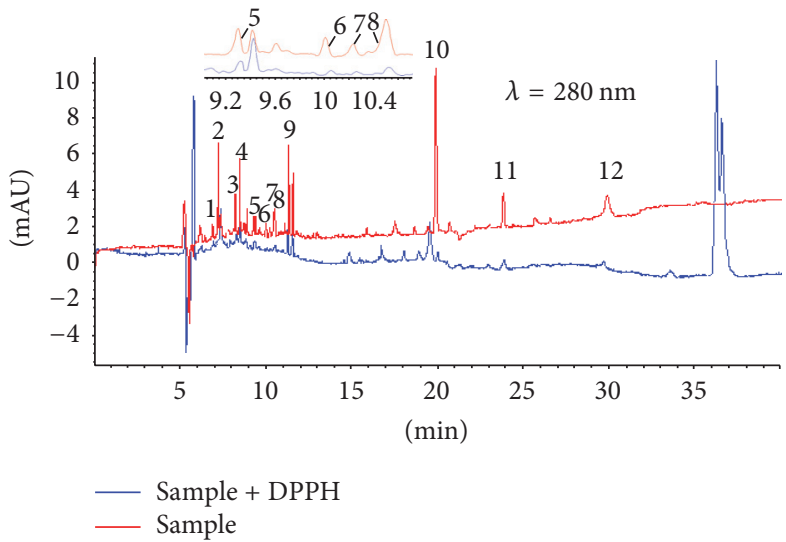

FIGURE 1: Schematic diagram of on-line DPPH-CE-DAD method.

screen antioxidants of Xuebijing injection, hydrodynamic injection was used for sample solution at 50 mbar for $8 \mathrm{~s}$ followed by DPPH solution at $50 \mathrm{mbar}$ pressure for $2 \mathrm{~s}$ (experimental group). Subsequently, a positive voltage was applied at $25 \mathrm{kV}$, with a capillary temperature of $22^{\circ} \mathrm{C}$. Analytes and DPPH were monitored at $280 \mathrm{~nm}$ and $517 \mathrm{~nm}$, respectively. Control group I was defined as Xuebijing injection on-line spiked methanol, and control group II was defined as DPPH solution on-line spiked deionized water.

Consequently, in comparison with control group II, the magnitude of decrease of DPPH peak seen in the electrophoretogram of the experimental group can be used to assess the antioxidant activity of the sample. Similarly, the magnitude of decrease of composition peak seen in the electrophoretogram of the experimental group can be used as a basis of antioxidant activity of Xuebijing injection when compared to control group I.

\section{Results and Discussion}

3.1. Optimization of On-Line DPPH-CE-DAD for Determination of Total Antioxidant Activity and Multicomponents of Xuebijing Injection. The electrophoresis system was optimized by adjusting the $\mathrm{pH}$ and concentrations of buffer, SDS, $\beta-\mathrm{CD}$, and organic modifier as well as voltage and cassette temperature. The running electrolyte for sample analysis was similar to that described previously [18], except that it was made possible to simultaneously separate and quantify DPPH and the antioxidants in one step by the newly DPPH-CEDAD method.

As is known, DPPH is not stable and would produce other substances in acid or alkaline conditions. The $\mathrm{pH}$ is the most significant variable influencing the performance of DPPH in comparison with other parameters. Hence, the $\mathrm{pH}$ were varied from 5.0 to 8.0 for sample separation while employing a running electrolyte comprising $20 \mathrm{mM} \mathrm{NaH}_{2} \mathrm{PO}_{4}, 100 \mathrm{mM}$ SDS, $10 \mathrm{mM} \beta-\mathrm{CD}$, and $5 \% \mathrm{ACN}(\mathrm{v} / \mathrm{v})$. When the $\mathrm{pH}$ was at 7,8 , and 9 , the peak shapes of all compounds were poor. At $\mathrm{pH}$ 5.0-6.0, acceptable separation was obtained for all the constituents and prolonged migration time was obtained with decreasing $\mathrm{pH}$ of the buffer, revealing that the migration velocity of weak electrolyte and the velocity of the electroosmotic flow (EOF) changed by regulating the $\mathrm{pH}$ [19]. From the results, $\mathrm{pH}$ of 5.5 was considered satisfactory with respect to migration time (Figure 2(a)).

Ionic strength or concentrations of buffer have significant effects on solute mobility and separation efficiency [20]. $\mathrm{NaH}_{2} \mathrm{PO}_{4}$ buffer at concentrations within the range of $0-40 \mathrm{mM}$ under constant instrumentation conditions was investigated. Comparing the concentration of $\mathrm{NaH}_{2} \mathrm{PO}_{4}$ buffer from 0 to $10 \mathrm{mM}$ which could not reach the baseline separation, 20-40 mM gave a good separation efficiency and resolution for each analyte. However, prolonged migration time was obtained at $30-40 \mathrm{mM}$ as shown in Figure 2(b). 


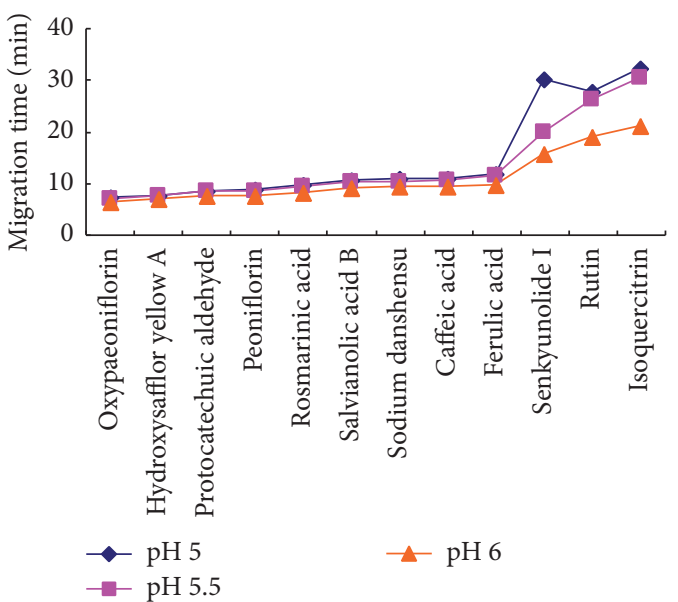

(a)

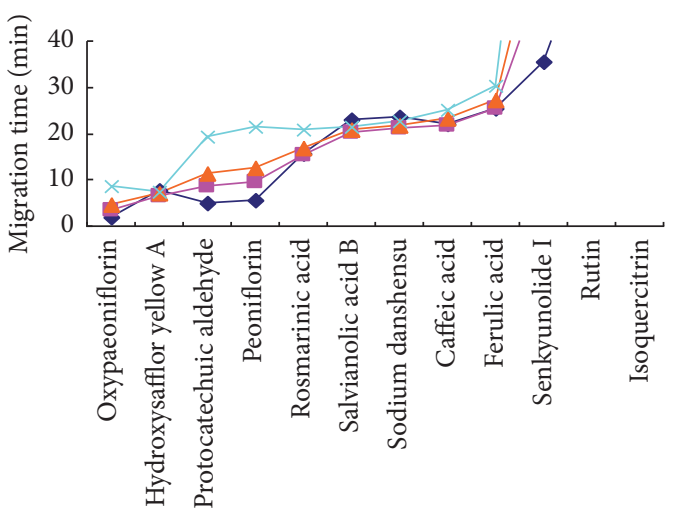

$\begin{array}{ll}\rightarrow \text { SDS } 50 \mathrm{mM} & \rightarrow \text { SDS } 100 \mathrm{mM} \\ \rightarrow \text { SDS } 80 \mathrm{mM} & \rightarrow \text { SDS } 150 \mathrm{mM}\end{array}$

(c)

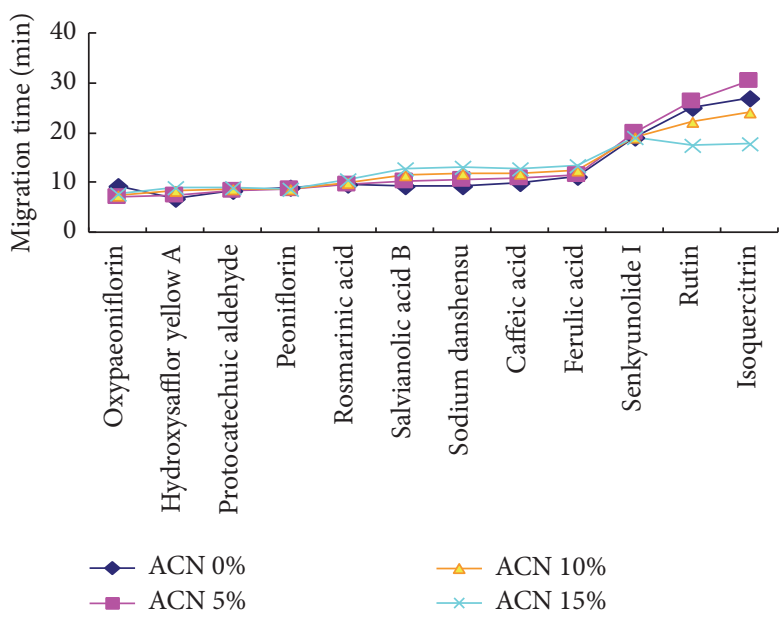

(e)

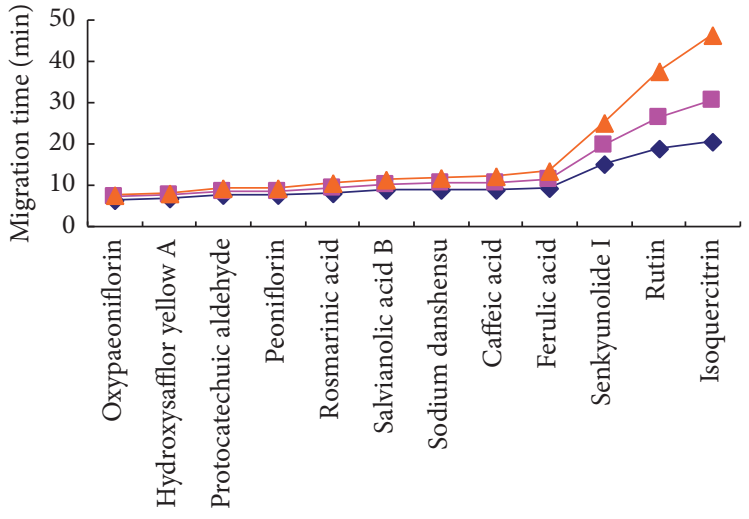

$\longrightarrow$ BGE $10 \mathrm{mM}$

BGE $20 \mathrm{mM}$

(b)

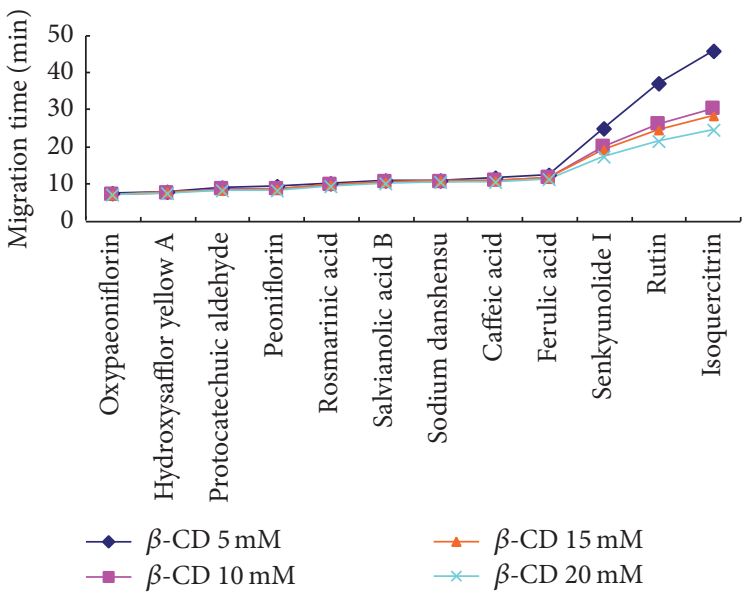

(d)

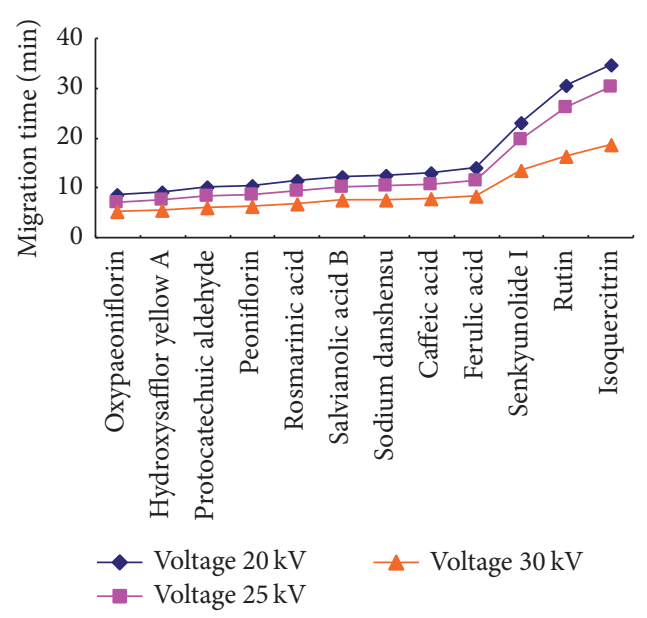

(f)

FIGURE 2: Effects of parameters on the migration time and resolution of twelve peaks: (a) pH of the phosphate buffer, (b) BGE concentration, (c) SDS concentration, (d) $\beta$-CD concentration, (e) acetonitrile concentration, and (f) separation voltage.

The $\mathrm{NaH}_{2} \mathrm{PO}_{4}$ buffer concentration for sample and DPPH determination was set at $20 \mathrm{mM}$.

The effects of SDS concentrations of $0,10,30,50,80$, 100 , and $150 \mathrm{mM}$ for sample separation were tested. As can be seen from Figure 2(c), prolonged migration time was obtained with increase in the concentration of SDS. At low SDS concentrations $(0-80 \mathrm{mM})$, the separation of protocatechuic aldehyde, peoniflorin, salvianolic acid B, sodium 
danshensu, and caffeic acid was not enough. Increasing the SDS concentration from 80 to $100 \mathrm{mM}$ led to a dramatic improvement in the resolution. When SDS concentration was raised substantially to $150 \mathrm{mM}$, the separation efficiency of rosmarinic acid and salvianolic acid B was poor as peoniflorin and sodium danshensu. Under $100 \mathrm{mM}$ SDS concentration, there was a good resolution among these compounds.

Addition of $\beta$-CD with different concentrations $(0,5$, 10,15 , and $20 \mathrm{mM}$ ) to the running electrolyte was studied. Slightly shortened migration time was observed with increase in the concentration of $\beta$-CD (Figure 2(d)). When the concentration of $\beta$-CD was $5 \mathrm{mM}$, the migration time of salvianolic acid B was the same as sodium danshensu. With the concentration of $10 \mathrm{mM} \beta-\mathrm{CD}$, a baseline resolution of each analyte was observed. Sodium danshensu partially overlapped with caffeic acid at 15 and $20 \mathrm{mM}$. It may be speculated that $\beta$-CD with a relative hydrophobic cavity forms inclusion complexes with compounds and the outer hydrophilic layer has been confirmed for the separation of analytes [20].

Organic solvent in the buffer could dissolve insoluble sample and alter hydrophobic analyte-micelle interactions by displacing the analytes from the micelle [21]. Acetonitrile was the most widely used organic modifier in $\mathrm{CE}$ [22]. Acetonitrile ranging from $0 \%$ to $15 \%$ was investigated. Figure 2(e) showed that the migration time of all analytes shortened when the percentage of acetonitrile added in the buffer was raised. The resolutions of salvianolic acid B and sodium danshensu generally tend to be good from $0 \%$ to $5 \%$. An extremely poor resolution resulted when the content of ACN was increased in the running electrolyte exceeding 5\%. Therefore, the optimum organic modifier was selected 5\% acetonitrile.

With these values established, voltages $(25-30 \mathrm{kV})$ and cassette temperatures $\left(20-25^{\circ} \mathrm{C}\right)$ which also influence the peak separation were investigated. A shorter migration time and poor resolution were obtained at a higher voltage $(30 \mathrm{kV})$, while a general increase of migration time was found at a lower voltage $(20 \mathrm{kV})$ (Figure 2(f)). Thus, a positive voltage of $25 \mathrm{kV}$ was chosen as the optimum separation voltage. The cassette temperature was finally set at $22^{\circ} \mathrm{C}$.

Based on our previous research [18], $20 \mathrm{mM} \mathrm{Na} \mathrm{NPO}_{4}$ $(\mathrm{pH} 6.0)+50 \mathrm{mM}$ SDS was the optimized condition for analyzing DPPH. However, an optimal condition comprising an electrolyte containing $20 \mathrm{mM} \mathrm{NaH}_{2} \mathrm{PO}_{4}$ (pH 5.5), $100 \mathrm{mM}$ SDS, $10 \mathrm{mM} \beta-\mathrm{CD}$, and $5 \% \mathrm{ACN}(\mathrm{v} / \mathrm{v})$ with the voltage and temperature setting at $25 \mathrm{kV}$ and $22^{\circ} \mathrm{C}$ was employed in all of our subsequent experiments, thereby reaching a balance between evaluating total antioxidant activity and screening antioxidants of Xuebijing injection in one step.

3.2. On-Line Determination of Total Antioxidant Activity of Sample. DPPH assay based on the reduction of absorbance at $517 \mathrm{~nm}$ of the stable DPPH radical by an antiradical is easy and potentially accurate for measuring the general radical scavenging capabilities of antioxidants [23]. Relying on the proposed DPPH-CE-DAD method as described above, the absorbance of DPPH on-line spiked Xuebijing injection

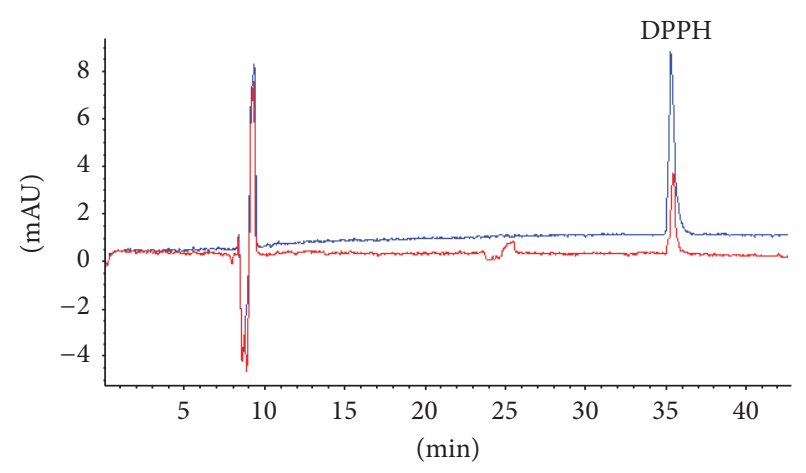

FIGURE 3: Capillary electropherograms of DPPH: DPPH (blue) and on-line mixed with Xuebijing injection (red). Experimental conditions: $50 \mu \mathrm{m}$ i.d. $\times 375 \mu \mathrm{m}$ o.d. $\times 60.5 \mathrm{~cm}$ length $(52 \mathrm{~cm}$ effective length), uncoated; $20 \mathrm{mM} \mathrm{NaH} \mathrm{PO}_{4}$ (pH 5.5), $100 \mathrm{mM}$ SDS, $10 \mathrm{mM} \beta$-CD, $5 \%(\mathrm{v} / \mathrm{v}) \mathrm{ACN}$, voltage, $25 \mathrm{kV}$; temperature, $22^{\circ} \mathrm{C}$; detection wavelength, $517 \mathrm{~nm}$; pressure injection, 50 mbar for $2 \mathrm{~s}$.

TABLE 1: The result of $\mathrm{IC}_{50}$ values of 10 batches of samples $(n=3)$.

\begin{tabular}{lc}
\hline Sample & $\mathrm{IC}_{50}(\mu \mathrm{g} / \mathrm{mL})$ \\
\hline 1301291 & 16.3 \\
1303041 & 14.5 \\
1303051 & 17.2 \\
1303071 & 16.6 \\
1302221 & 9.40 \\
1302231 & 16.8 \\
1303021 & 17.7 \\
1303042 & 11.7 \\
1303031 & 6.70 \\
1302051 & 7.40 \\
\hline
\end{tabular}

(experimental group) was relatively weak compared to that of the on-line spiked deionized water (control group I) because DPPH would react with antioxidants of Xuebijing injection in the capillary after injecting separately (Figure 3). The relative percentage of inhibition of DPPH was determined by the following equation: [Inhibition $(\%)=\left(P_{0}-P_{1}\right) / P_{0} \times 100 \%$ ]. $P_{0}$ was peak area of DPPH (on-line spiked deionized water) and $P_{1}$ was peak area of DPPH (on-line spiked Xuebijing injection). In the total antioxidant activity assay of samples, Xuebijing injections with different diluted times were on-line spiked with DPPH, respectively, to carry out the maximal inhibitory concentration at $50 \%\left(\mathrm{IC}_{50}\right)$ which denotes the diluted times of sample required to scavenge $50 \%$ of DPPH radicals. Table 1 displayed the $\mathrm{IC}_{50}$ values of 10 batches of Xuebijing injections. Results illustrated that Xuebijing injections have antioxidant activity and there was a significant difference in the $\mathrm{IC}_{50}$ values of each batch which could speculate that the discrimination of activity was as a result of different contents of antioxidants.

3.3. Method Validation. The calibration graphs of twelve antioxidants of Xuebijing injection were established with the peak area ratio as ordinate $(y)$ versus the concentration 
TABLE 2: The calibration curves, linearity ranges, LODs, LOQs, and recoveries of twelve compounds $(n=6)$.

\begin{tabular}{lcccccc}
\hline Compounds & Regression equation & $R^{2}$ & $\begin{array}{c}\text { Linearity range } \\
(\mu \mathrm{g} / \mathrm{mL})\end{array}$ & $\begin{array}{c}\text { LOD } \\
(\mu \mathrm{g} / \mathrm{mL})\end{array}$ & $\begin{array}{c}\text { LOQ } \\
(\mu \mathrm{g} / \mathrm{mL})\end{array}$ & $\begin{array}{c}\text { Recovery } \\
\text { Average }(\%)\end{array}$ \\
RSD $(\%)$
\end{tabular}

in $\mu \mathrm{g} / \mathrm{mL}$ as abscissa $(x)$. They were obtained over the range of $33.3-400 \mu \mathrm{g} / \mathrm{mL}, \quad 25-600 \mu \mathrm{g} / \mathrm{mL}, \quad 4.2-50 \mu \mathrm{g} / \mathrm{mL}, \quad 62.5-$ $2000 \mu \mathrm{g} / \mathrm{mL}, \quad 5.8-70 \mu \mathrm{g} / \mathrm{mL}, \quad 10-120 \mu \mathrm{g} / \mathrm{mL}, 13-156 \mu \mathrm{g} / \mathrm{mL}$, $2.5-30 \mu \mathrm{g} / \mathrm{mL}, 2.5-60 \mu \mathrm{g} / \mathrm{mL}, 20-480 \mu \mathrm{g} / \mathrm{mL}, 13-156 \mu \mathrm{g} / \mathrm{mL}$, and $11-132 \mu \mathrm{g} / \mathrm{mL}$ for oxypaeoniflorin, hydroxysafflor yellow A, protocatechuic aldehyde, peoniflorin, rosmarinic acid, salvianolic acid B, sodium danshensu, caffeic acid, ferulic acid, senkyunolide I, rutin, and isoquercitrin, respectively. The straight lines of the twelve compounds obtained from six separate experiments had good correlation coefficients. The regression equations and their correlation coefficients were $y=0.0658 x+0.3049\left(R^{2}=0.9991\right), y=0.3099 x-1.1776$ $\left(R^{2}=0.9999\right), y=0.6055 x-0.2398\left(R^{2}=0.9996\right), y=$ $0.1948 x-0.6145\left(R^{2}=0.9998\right), y=0.3097 x-0.0843\left(R^{2}=\right.$ $0.9990), y=0.2089 x-0.526\left(R^{2}=0.9995\right), y=0.1248 x-$ $0.311\left(R^{2}=0.9982\right), y=0.8707 x-0.2758\left(R^{2}=0.9995\right), y=$ $0.8666 x-0.3651\left(R^{2}=0.9995\right), y=0.198 x-0.8145\left(R^{2}=\right.$ $0.9993), y=0.2938 x-1.0819\left(R^{2}=0.9990\right), y=0.3888 x-$ $0.0695\left(R^{2}=0.9991\right)$, respectively. The limit of detection (LOD) and the limit of quantitation (LOQ) were described as the sample concentrations gave rise to a signal-to-noise $(\mathrm{S} / \mathrm{N})$ ratio of 3 and 10 , respectively. Table 2 showed the LODs and LOQs of oxypaeoniflorin, hydroxysafflor yellow A, protocatechuic aldehyde, peoniflorin, rosmarinic acid, salvianolic acid B, sodium danshensu, caffeic acid, ferulic acid, senkyunolide I, rutin, and isoquercitrin.

The precision and accuracy of the proposed method was evaluated on the basis of peak area expressed as relative standard deviation (RSD). The intraday and interday precisions at three typical assay concentrations of each constituent and DPPH were evaluated for six replicates within one day and over three successive days, respectively. The intra- and interday accuracies of twelve antioxidants were within the range of $92.1 \%-105 \%$. The RSDs of mean precision of twelve antioxidants were below $5.1 \%$ for intraday and $4.9 \%$ for interday, respectively (Table 3). The RSDs for both intraand interday of DPPH were $2.4 \%$ and $0.9 \%$, respectively.
The recoveries of each component were calculated from the calibration graph constructed from the sample spiked with an equivalent amount of the standard solution. The results of recoveries of twelve compounds listed in Table 2 were all in the range of $97.4 \%-102 \%$ and the RSDs were below $4.8 \%$.

The stability expressed by peak area was evaluated by injecting repetitive quality control samples of twelve compounds at low, medium, and high concentrations and DPPH in the CE equipment over $24 \mathrm{~h}$. Table 3 showed that the accuracies of antioxidants were within the range of $94.3 \%-$ $104 \%$ and the RSDs of all compounds and DPPH were below $8.5 \%$, respectively. The result confirmed that they were stable for $24 \mathrm{~h}$ at $4^{\circ} \mathrm{C}$.

All these data indicated that electrophoretic assay method was acceptable and could be applied in determining the total antioxidant activity of the multicomponents of Xuebijing injection.

3.4. On-Line Screening of Antioxidants of Sample. Although the antioxidant activity of Xuebijing injection has been demonstrated, it was not clear as to which component was responsible for the antioxidant activity. Taking the identification of active ingredients into account, the peak area of all of the ingredients of Xuebijing injection was compared successively between sample on-line spiked DPPH (experimental group) and sample on-line spiked methanol (control group II) (Figure 4). The components examined from the decreased peak area were compared with reference standard to facilitate the identification and confirmation of the antioxidants of Xuebijing injection, including oxypaeoniflorin, hydroxysafflor yellow A, protocatechuic aldehyde, peoniflorin, rosmarinic acid, salvianolic acid B, sodium danshensu, caffeic acid, ferulic acid, senkyunolide I, rutin, and isoquercitrin (Figure 4). The newly on-line DPPH-CE-DAD method facilitated the rapid screening of major antioxidants of TCMs and was meaningful for further quality assessment using key selected markers. 
TABLE 3: Intraday and interday accuracy and precision and stability of twelve compounds and DPPH $(n=6)$.

\begin{tabular}{|c|c|c|c|c|c|c|c|}
\hline \multirow{2}{*}{ Compounds } & \multirow{2}{*}{ Concentrations $(\mu \mathrm{g} / \mathrm{mL})$} & \multicolumn{2}{|c|}{ Intraday } & \multicolumn{2}{|c|}{ Interday } & \multicolumn{2}{|c|}{ Stability for $24 \mathrm{~h}$} \\
\hline & & Accuracy (\%) & RSD (\%) & Accuracy (\%) & RSD (\%) & Remains (\%) & RSD (\%) \\
\hline \multirow{3}{*}{ Oxypaeoniflorin } & 66.7 & 94.9 & 3.4 & 97.8 & 2.8 & 97.2 & 3.7 \\
\hline & 133.3 & 97.6 & 2.3 & 97.5 & 0.5 & 99.9 & 3.4 \\
\hline & 400 & 96.6 & 2.7 & 98.1 & 1.4 & 96.5 & 0.9 \\
\hline \multirow{3}{*}{ Hydroxysafflor yellow A } & 50 & 97.8 & 1.1 & 97.5 & 0.2 & 100.9 & 4.5 \\
\hline & 100 & 92.1 & 4.1 & 97.0 & 4.9 & 95.0 & 4.7 \\
\hline & 300 & 96.7 & 5.1 & 98.4 & 2.3 & 97.1 & 0.8 \\
\hline \multirow{3}{*}{ Protocatechuic aldehyde } & 8.3 & 103.7 & 4.9 & 97.8 & 4.9 & 97.4 & 8.5 \\
\hline & 16.7 & 97.4 & 2.6 & 100.3 & 2.8 & 99.4 & 3.0 \\
\hline & 50 & 100.6 & 0.9 & 98.5 & 3.1 & 101.3 & 0.9 \\
\hline \multirow{3}{*}{ Peoniflorin } & 166.7 & 102.1 & 0.4 & 98.7 & 3.0 & 101.9 & 0.3 \\
\hline & 333.3 & 100.6 & 0.8 & 98.6 & 1.9 & 101.5 & 1.3 \\
\hline & 1000 & 101.8 & 0.2 & 99.2 & 2.7 & 103.4 & 2.2 \\
\hline \multirow{3}{*}{ Rosmarinic acid } & 11.7 & 95.8 & 3.7 & 98.8 & 4.5 & 95.6 & 0.2 \\
\hline & 23.3 & 102.2 & 3.5 & 102.8 & 1.5 & 102.6 & 0.6 \\
\hline & 70 & 101.9 & 3.0 & 102.3 & 0.4 & 101.9 & 0.6 \\
\hline \multirow{3}{*}{ Salvianolic acid B } & 20 & 96.5 & 4.0 & 98.2 & 1.7 & 97.7 & 2.0 \\
\hline & 40 & 98.7 & 2.9 & 96.1 & 2.2 & 99.2 & 0.8 \\
\hline & 120 & 98.4 & 4.0 & 96.7 & 4.1 & 98.0 & 0.4 \\
\hline \multirow{3}{*}{ Sodium danshensu } & 26 & 101.6 & 4.4 & 101.7 & 0.2 & 103.6 & 2.9 \\
\hline & 52 & 104.7 & 2.4 & 104.2 & 1.3 & 103.7 & 1.3 \\
\hline & 156 & 102.9 & 3.6 & 101.5 & 1.1 & 102.1 & 1.0 \\
\hline \multirow{3}{*}{ Caffeic acid } & 5 & 105.2 & 4.5 & 99.5 & 4.6 & 103.9 & 1.5 \\
\hline & 10 & 100.2 & 2.8 & 96.5 & 3.6 & 98.9 & 1.8 \\
\hline & 30 & 97.8 & 2.8 & 97.2 & 1.2 & 97.7 & 0.3 \\
\hline \multirow{3}{*}{ Ferulic acid } & 5 & 102.0 & 4.7 & 100.4 & 3.7 & 100.3 & 2.0 \\
\hline & 10 & 100.7 & 2.8 & 97.7 & 3.1 & 101.9 & 1.8 \\
\hline & 30 & 94.4 & 1.7 & 97.0 & 2.5 & 97.4 & 4.5 \\
\hline \multirow{3}{*}{ Senkyunolide I } & 40 & 99.2 & 3.9 & 100.3 & 2.8 & 97.9 & 1.7 \\
\hline & 80 & 101.4 & 2.3 & 100.2 & 1.3 & 101.7 & 0.4 \\
\hline & 240 & 100.7 & 0.8 & 101.8 & 0.9 & 101.2 & 0.7 \\
\hline \multirow{3}{*}{ Rutin } & 26 & 103.0 & 3.6 & 101.3 & 1.4 & 100.1 & 3.8 \\
\hline & 52 & 97.0 & 1.5 & 97.8 & 0.8 & 94.3 & 4.0 \\
\hline & 156 & 99.7 & 2.2 & 97.5 & 2.5 & 99.5 & 0.3 \\
\hline \multirow{3}{*}{ Isoquercitrin } & 22 & 100.0 & 3.3 & 97.4 & 2.2 & 97.8 & 3.0 \\
\hline & 44 & 100.7 & 4.9 & 100.2 & 1.3 & 99.8 & 1.0 \\
\hline & 132 & 104.2 & 1.7 & 100.6 & 3.6 & 101.4 & 3.7 \\
\hline DPPH & 1000 & - & 2.4 & - & 0.9 & - & 1.4 \\
\hline
\end{tabular}

3.5. Sample Analysis. The developed DPPH-CE-DAD method was applied for analyzing the contents of twelve antioxidants of 10 batches of Xuebijing injection under the optimized conditions. Figure 5 displayed the typical chromatographic profile of the sample. The results of the sample analysis for oxypaeoniflorin, hydroxysafflor yellow A, protocatechuic aldehyde, peoniflorin, rosmarinic acid, salvianolic acid B, sodium danshensu, caffeic acid, ferulic acid, senkyunolide $\mathrm{I}$, rutin, and isoquercitrin were in the range of $39.1-54.6 \mu \mathrm{g} / \mathrm{mL}, 381.2-529.4 \mu \mathrm{g} / \mathrm{mL}$, $15.4-27.3 \mu \mathrm{g} / \mathrm{mL}, \quad 1736.6-1980.9 \mu \mathrm{g} / \mathrm{mL}, \quad 10.8-25.4 \mu \mathrm{g} / \mathrm{mL}$,
6.9-17.4 $\mu \mathrm{g} / \mathrm{mL}, \quad 13.1-24.7 \mu \mathrm{g} / \mathrm{mL}, \quad 9.4-14.6 \mu \mathrm{g} / \mathrm{mL}, \quad 32.0-$ $37.0 \mu \mathrm{g} / \mathrm{mL}, 266.1-382.4 \mu \mathrm{g} / \mathrm{mL}, 18.8-28.0 \mu \mathrm{g} / \mathrm{mL}$, and $26.7-$ $40.2 \mu \mathrm{g} / \mathrm{mL}$, respectively, which illustrated major difference in the concentrations of twelve antioxidants of each batch of Xuebijing injection (Table 4).

Given the difference in the $\mathrm{IC}_{50}$ values of 10 batches, it was likely to be as a result of the different contents of antioxidants. As shown in Figure 6, the data from total content of twelve compounds with antioxidant activity across 10 batches of Xuebijing injections showed a good correlation $\left(R^{2}=0.931\right)$ with above-referred data of determination of 


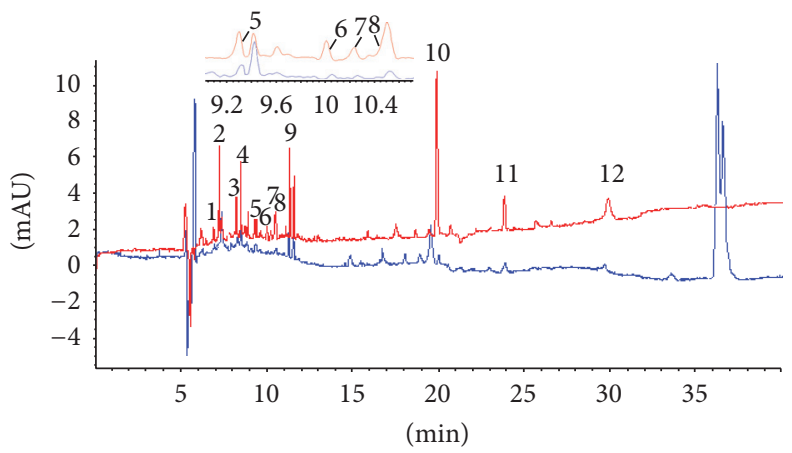

FIgURE 4: Capillary electropherograms of Xuebijing injection: Xuebijing injection (red) and on-line mixed with DPPH (blue). Peaks: $1=$ oxypaeoniflorin, 2 = hydroxysafflor yellow A, 3 = protocatechuic aldehyde, $4=$ peoniflorin, $5=$ rosmarinic acid, $6=$ salvianolic acid $\mathrm{B}, 7=$ sodium danshensu, $8=$ caffeic acid, $9=$ ferulic acid, $10=$ senkyunolide I, $11=$ rutin, and $12=$ isoquercitrin. Experimental conditions: $50 \mu \mathrm{m}$ i.d. $\times 375 \mu \mathrm{m}$ o.d. $\times 60.5 \mathrm{~cm}$ length (52 cm effective length), uncoated; $20 \mathrm{mM} \mathrm{NaH}_{2} \mathrm{PO}_{4}$ (pH 5.5), $100 \mathrm{mM} \mathrm{SDS}, 10 \mathrm{mM} \beta-\mathrm{CD}, 5 \%$ (v/v) ACN, voltage, $25 \mathrm{kV}$; temperature, $22^{\circ} \mathrm{C}$; detection wavelength, $280 \mathrm{~nm}$; pressure injection, $50 \mathrm{mbar}$ for $8 \mathrm{~s}$.

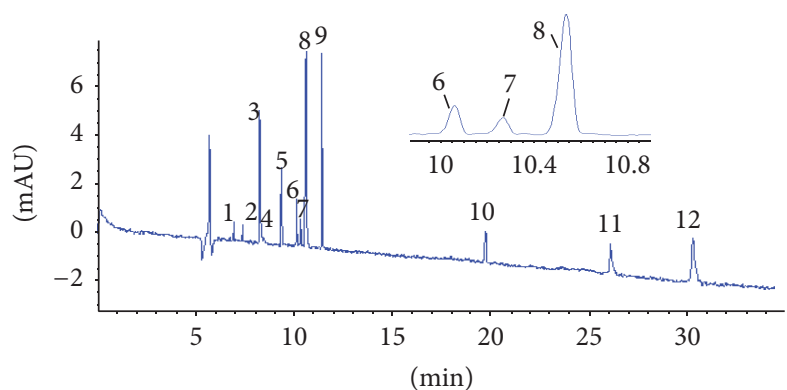

(a)

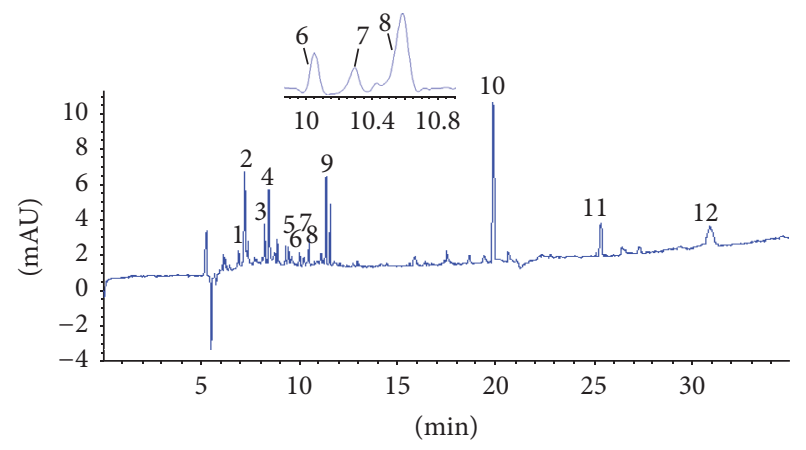

(b)

FIGURE 5: Capillary electropherograms of standard mixture of twelve compounds (a) and Xuebijing injection (b). Peaks: $1=$ oxypaeoniflorin, 2 = hydroxysafflor yellow A, $3=$ protocatechuic aldehyde, $4=$ peoniflorin, $5=$ rosmarinic acid, $6=$ salvianolic acid B, $7=$ sodium danshensu, 8 $=$ caffeic acid, $9=$ ferulic acid, $10=$ senkyunolide I, $11=$ rutin, $12=$ isoquercitrin. Experimental conditions: $50 \mu \mathrm{m} \mathrm{i.d.} \times 375 \mu \mathrm{m}$ o.d. $\times 60.5 \mathrm{~cm}$ length (52 cm effective length), uncoated; $20 \mathrm{mM} \mathrm{NaH}_{2} \mathrm{PO}_{4}$ (pH 5.5), $100 \mathrm{mM} \mathrm{SDS}, 10 \mathrm{mM} \beta$-CD, 5\% (v/v) ACN, $25 \mathrm{kV}$; temperature, $22^{\circ} \mathrm{C}$; detection wavelength, $280 \mathrm{~nm}$; pressure injection, 50 mbar for $8 \mathrm{~s}$.

total antioxidant activity of samples. The result illustrated that twelve selected antioxidants can serve as key quality control markers to maintain batch-to-batch uniformity and efficacy. It was also validated that the proposed method was feasible and accurate in determining the total antioxidant activity with multiple active ingredients for quality control of TCMs.

\section{Conclusions}

To improve the previous DPPH-CE-DAD method, we developed a simple method that made it possible to separate and quantify DPPH and the antioxidants under the same condition. In the course of on-line mixing DPPH and sample, the magnitude of decrease of DPPH peak seen in the electrophoretogram could calculate the total antioxidant activity of Xuebijing injection. Similarly, twelve antioxidant active ingredients containing oxypaeoniflorin, hydroxysafflor yellow A, protocatechuic aldehyde, peoniflorin, rosmarinic acid, salvianolic acid B, sodium danshensu, caffeic acid, ferulic acid, senkyunolide I, rutin, and isoquercitrin were

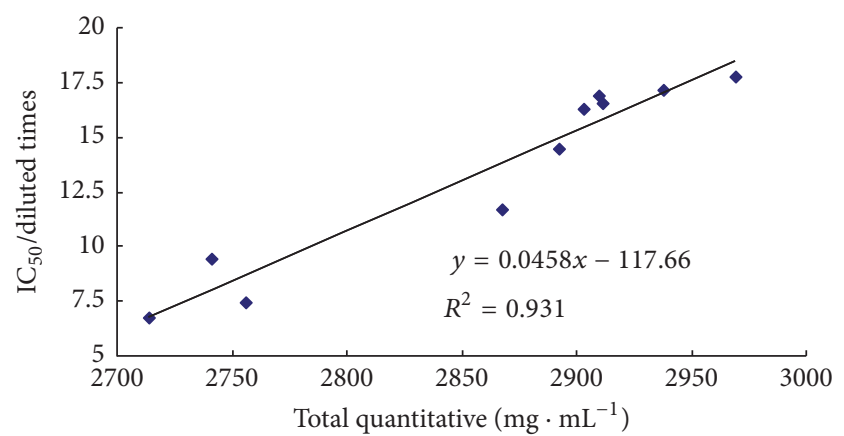

FIGURE 6: Relationship of the total quantitative and antioxidant activity of Xuebijing injection.

rapidly screened relying on the magnitude of decrease of composition peak seen. Results illustrated that the advantages of this developed method were reagent-saving, rapid, feasible, and green technique which is not harmful to the environment. The improved on-line DPPH-CE-DAD method 


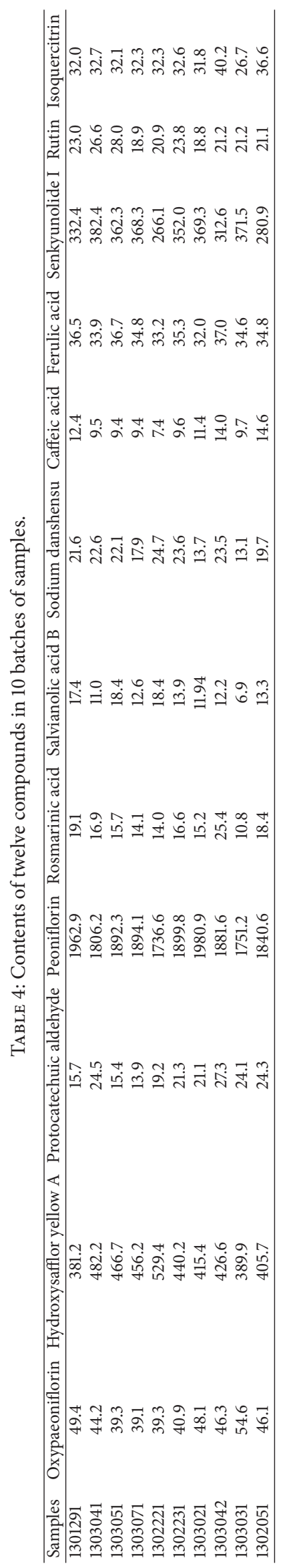


achieved the goal in analyzing the total antioxidant activity and contents of antioxidants of Xuebijing injection in one step and was applicable to evaluate the quality of TCMs which exerted directly their antioxidant effects on the radical itself. Furthermore, this presented approach can be applied in evaluating the antioxidant activity of unknown molecules.

\section{Competing Interests}

The authors have declared that there are no competing interests regarding the publication of this paper.

\section{Authors' Contributions}

Jiao Liu and first author Jin Li contributed equally to this study.

\section{Acknowledgments}

This research was supported by National Natural Science Foundation of China (81503213 and 81303140), Stated Key Development Program for Basic Research of China (no. 973:2012CB723504), and the State Science \& Technology Commission of MOST of China (2014ZX09304307-001-005 and 2014ZX09201022-004).

\section{References}

[1] M. W. Liu, Y. H. Wang, C. Y. Qian, and H. Li, "Xuebijing exerts protective effects on lung permeability leakage and lung injury by upregulating Toll-interacting protein expression in rats with sepsis," International Journal of Molecular Medicine, vol. 34, no. 6, pp. 1492-1504, 2014.

[2] H. Wisplinghoff, T. Bischoff, S. M. Tallent, H. Seifert, R. P. Wenzel, and M. B. Edmond, "Nosocomial bloodstream infections in US hospitals: analysis of 24,179 cases from a prospective nationwide surveillance study," Clinical Infectious Diseases, vol. 39, no. 3, pp. 309-317, 2004.

[3] X.-D. He, Y. Wang, Q. Wu et al., "Xuebijing protects rats from sepsis challenged with Acinetobacter baumannii by promoting annexin Al expression and inhibiting proinflammatory cytokines secretion," Evidence-Based Complementary and Alternative Medicine, vol. 2013, Article ID 804940, 11 pages, 2013.

[4] D. G. Li, L. Lu, J. L. Zhang et al., "Mitigating the effects of Xuebijing Injection on hematopoietic cell injury induced by total body irradiation with $\gamma$ rays by decreasing reactive oxygen species levels," International Journal of Molecular Sciences, vol. 15, no. 6, pp. 10541-10553, 2014.

[5] Q. Yin and C. S. Li, "Treatment effects of xuebijing injection in severe septic patients with disseminated intravascular coagulation," Evidence-Based Complementary and Alternative Medicine, vol. 2014, Article ID 949254, 6 pages, 2014.

[6] Y. Guo, Y. Wang, X. Huang et al., "Determination of hydroxysafflor yellow A in biological fluids of patients with traumatic brain injury by UPLC-ESI-MS/MS after injection of xuebijing," Biomedical Chromatography, vol. 28, no. 8, pp. 1090-1095, 2014.

[7] C. Deblanc, F. Robert, T. Pinard et al., "Pre-infection of pigs with Mycoplasma hyopneumoniae induces oxidative stress that influences outcomes of a subsequent infection with a swine influenza virus of H1N1 subtype," Veterinary Microbiology, vol. 162, no. 2-4, pp. 643-651, 2013.

[8] S. Kelekçi, O. Evliyaoğlu, V. Sen et al., "The relationships between clinical outcome and the levels of total antioxidant capacity (TAC) and coenzyme Q (CoQ10) in children with pandemic influenza (H1N1) and seasonal flu," European Review for Medical and Pharmacological Sciences, vol. 16, no. 8, pp. 1033-1038, 2012.

[9] K. Zhai, G. Gao, W. Cao, L. Zhao, X. Fang, and H. Duan, "Simultaneous HPLC determination of four active compounds in fengshiding capsules, a Chinese medicine," Indian Journal of Pharmaceutical Sciences, vol. 76, no. 5, pp. 445-449, 2014.

[10] S. Jiang, P. Lai, J. Li, and G. Wang, "Antioxidant activities and essential oil composition of Herba Artemisiae Scopariae from China," Journal of Oleo Science, vol. 61, no. 5, pp. 291-295, 2012.

[11] S. Xiao, R. R. Yu, N. Ai, and X. H. Fan, "Rapid screening natural-origin lipase inhibitors from hypolipidemic decoctions by ultrafiltration combined with liquid chromatography-mass spectrometry," Journal of Pharmaceutical and Biomedical Analysis, vol. 104, pp. 67-74, 2015.

[12] H.-T. Liu, Y.-F. Wang, O. Olaleye et al., "Characterization of in vivo antioxidant constituents and dual-standard quality assessment of Danhong injection," Biomedical Chromatography, vol. 27, no. 5, pp. 655-663, 2013.

[13] R. G. de Oliveira Jr., G. R. Souza, A. L. Guimarães et al., "Dried extracts of Encholirium spectabile (Bromeliaceae) present antioxidant and photoprotective activities in vitro," Journal of Young Pharmacists, vol. 5, no. 3, pp. 102-105, 2013.

[14] H. A. G. Niederländer, T. A. van Beek, A. Bartasiute, and I. I. Koleva, "Antioxidant activity assays on-line with liquid chromatography," Journal of Chromatography A, vol. 1210, no. 2, pp. 121-134, 2008.

[15] S. Agatonovic-Kustrin, D. Babazadeh Ortakand, D. W. Morton, and A. P. Yusof, "Rapid evaluation and comparison of natural products and antioxidant activity in calendula, feverfew, and German chamomile extracts," Journal of Chromatography A, vol. 1385, pp. 103-110, 2015.

[16] Y. Zhang, S. Shi, Y. Wang, and K. Huang, "Target-guided isolation and purification of antioxidants from Selaginella sinensis by offline coupling of DPPH-HPLC and HSCCC experiments," Journal of Chromatography B: Biomedical Sciences and Applications, vol. 879, no. 2, pp. 191-196, 2011.

[17] S. Vshivkov, E. Pshenichnov, Z. Golubenko, A. Akhunov, S. Namazov, and R. D. Stipanovic, "Capillary electrophoresis to quantitate gossypol enantiomers in cotton flower petals and seed," Journal of Chromatography B: Analytical Technologies in the Biomedical and Life Sciences, vol. 908, pp. 94-97, 2012.

[18] Y. Chang, J. Liu, Y. Bai et al., “The activity-integrated method for quality assessment of reduning injection by on-line DPPHCE-DAD," PLoS ONE, vol. 9, no. 9, Article ID e106254, 2014.

[19] S. Orlandini, R. Gotti, and S. Furlanetto, "Multivariate optimization of capillary electrophoresis methods: a critical review," Journal of Pharmaceutical and Biomedical Analysis, vol. 87, pp. 290-307, 2014.

[20] H. H. Yeh, Y. H. Yang, J. Y. Ko, and S. H. Chen, "Rapid determination of piracetam in human plasma and cerebrospinal fluid by micellar electrokinetic chromatography with sample direct injection," Journal of Chromatography A, vol. 1120, no. 1-2, pp. 27-34, 2006.

[21] F. K. Liu, "Preconcentration and separation of neutral steroid analytes using a combination of sweeping micellar electrokinetic chromatography and a Au nanoparticle-coated solid phase 
extraction sorbent," Journal of Chromatography A, vol. 1215, no. 1-2, pp. 194-202, 2008.

[22] R. Gottardo, A. Bertaso, J. Pascali et al., "Micellar electrokinetic chromatography: a new simple tool for the analysis of synthetic cannabinoids in herbal blends and for the rapid estimation of their $\log P$ values," Journal of Chromatography A, vol. 1267, pp. 198-205, 2012.

[23] O. A. Chat, M. H. Najar, M. A. Mir, G. M. Rather, and A. A. Dar, "Effects of surfactant micelles on solubilization and DPPH radical scavenging activity of Rutin," Journal of Colloid and Interface Science, vol. 355, no. 1, pp. 140-149, 2011. 


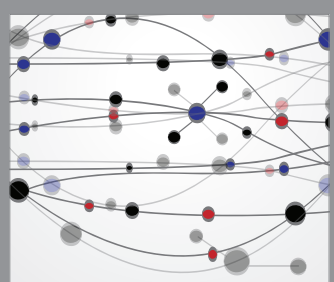

The Scientific World Journal
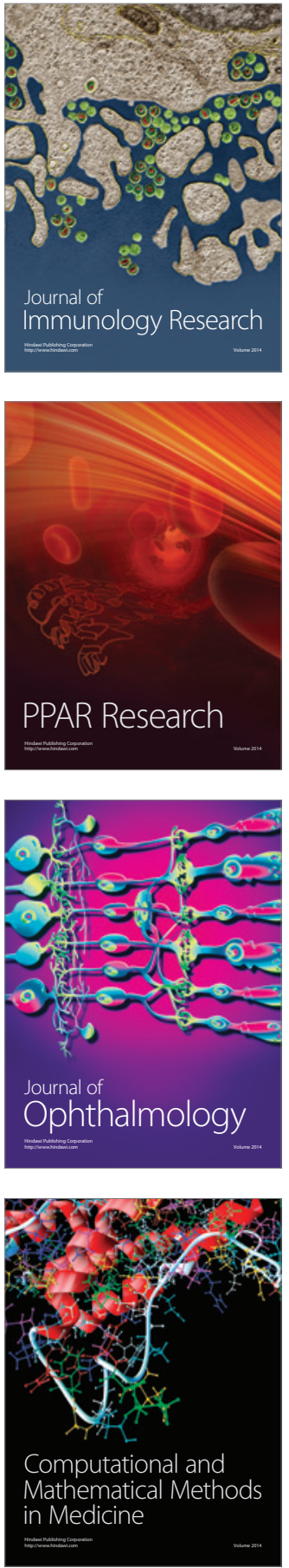

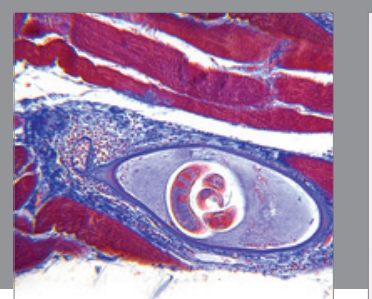

Gastroenterology Research and Practice

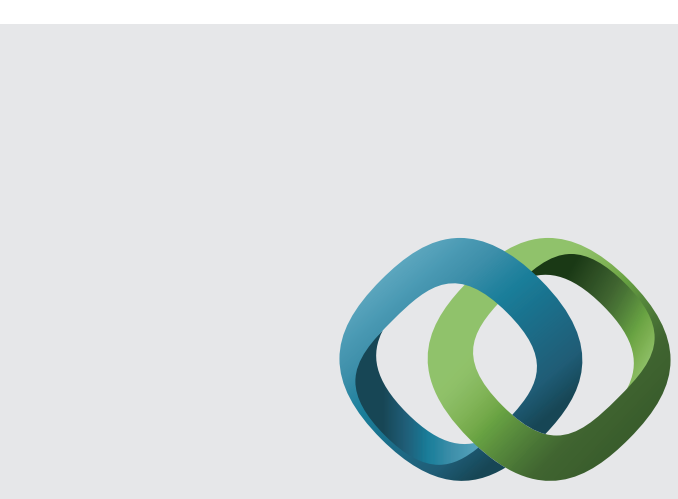

\section{Hindawi}

Submit your manuscripts at

http://www.hindawi.com
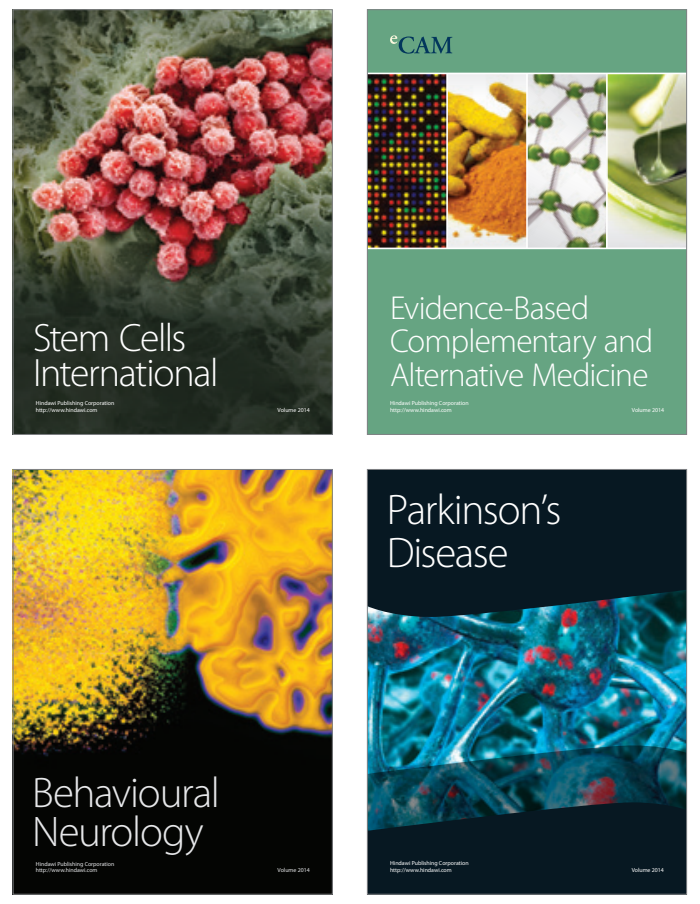
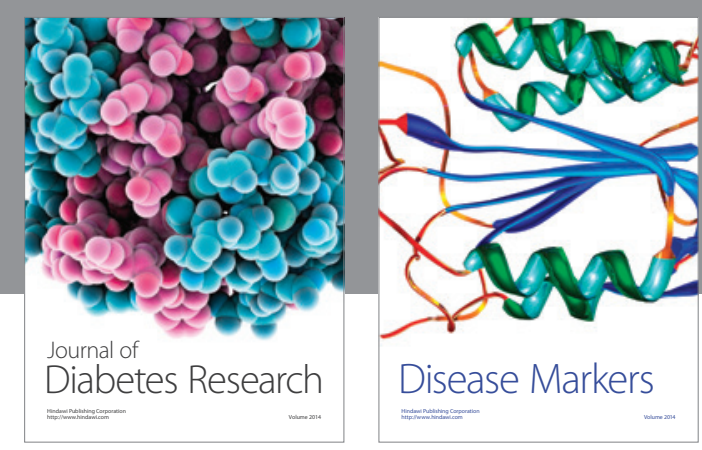

Disease Markers
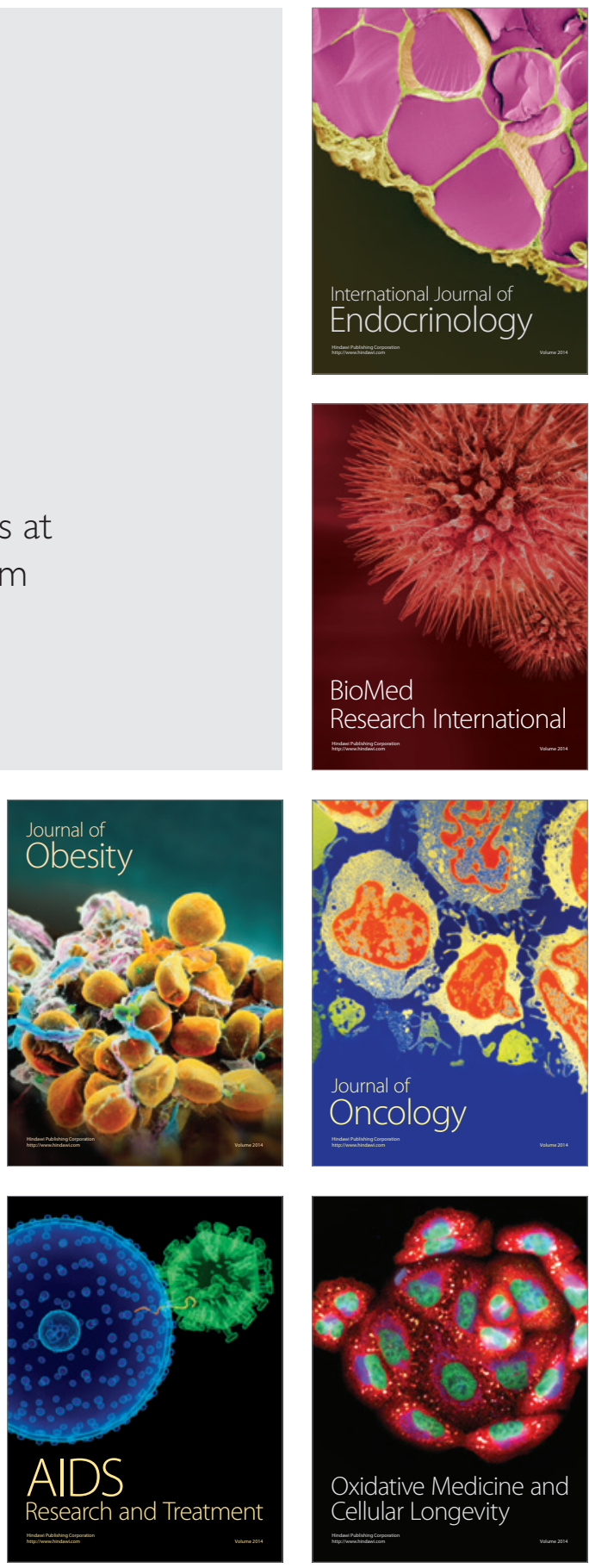